\title{
Produção e partição da biomassa de Desmodium incanum em resposta à aplicação de fósforo(1)
}

\author{
Noemia Corsini Silva( ${ }^{(2)}$, Lucia Brandão Franke ${ }^{(2)}$, Carlos Nabinger(2) e Ricardo Barreto(3)
}

\begin{abstract}
Resumo - Este trabalho teve o objetivo de analisar o efeito do tipo de solo e de doses de fósforo (P) sobre a produção e partição da biomassa de Desmodium incanum DC. Foram feitas quatro avaliações ao longo do tempo, visando determinar a evolução da biomassa total e sua partição em parte aérea e radicular. Os tratamentos consistiram de dois tipos de solo (Plintossolo e Latossolo) e quatro doses de $\mathrm{P}\left(0,30,60,90 \mathrm{mg}\right.$ de $\mathrm{P}_{2} \mathrm{O}_{5} / \mathrm{kg}$ de solo). Foi utilizado o delineamento completamente casualizado, num fatorial $4 \times 2$, com quatro repetições. Determinou-se o teor total de $\mathrm{N}$ e P na matéria seca da parte aérea. Na ausência da adubação fosfatada, os dois solos apresentaram potencial semelhante para a produção de matéria seca. O Latossolo, quando adubado, foi superior ao Plintossolo. A deficiência de $\mathrm{P}$ e a menor fertilidade natural do solo alterou a partição da biomassa. A alocação de assimilados para a parte aérea foi prejudicada em favor do crescimento radicular. Modelos de diluição de $\mathrm{P}$ e $\mathrm{N}$ foram testados, propondo-se valores de parâmetros para plantas individuais.
\end{abstract}

Termos para indexação: leguminosa, morfogênese, matéria seca, adubação fosfatada.

Biomass production and partitioning of Desmodium incanum in response to phosphate application

Abstract - This work had the objective of analysing the effect of soil type and phosphate (P) levels on production and biomass partition of Desmodium incanum DC. Four evaluations aiming at determining the evolution of total biomass and root and shoot biomass partition were made during the period. The treatments were two types of soil (Plinthaquult and Hapludox) and four levels of phosphate $(0,30,60$, $90 \mathrm{mg}$ of $\mathrm{P}_{2} \mathrm{O}_{5} / \mathrm{kg}$ of soil) arranged in a completely randomized design, with four replications. The total $\mathrm{N}$ and $\mathrm{P}$ content was determined in the dry matter. In the absence of $\mathrm{P}$ fertilization both soils had a similar potential for dry matter production. When fertilized, the Hapludox was superior to the Plinthaquult. $\mathrm{P}$ deficiency and lower soil natural fertility altered biomass partition. The allocation of assimilates to plant aerial parts was smaller, favoring root growth. Models for P and $\mathrm{N}$ dilution were tested, and parameter values for individual plants were proposed

Index terms: leguminosae, morphogenesis, dry matter, phosphate fertilizers.

\section{Introdução}

A partição de assimilados compreende a alocação e a distribuição ou o transporte de assimilados de carbono (moléculas ou íons) para locais de consumo

(1) Aceito para publicação em 6 de junho de 2000

Extraído da dissertação de mestrado, apresentada pelo primeiro autor à Universidade Federal do Rio Grande do Sul (UFRGS), Porto Alegre, RS.

(2)UFRGS, Faculdade de Agronomia, Dep. de Plantas Forrageiras e Agrometeorologia, Caixa Postal 776, CEP 91501-970 Porto Alegre, RS. E-mail: 1bfranke@vortex.ufrgs.br, nabinger@vortex.ufrgs.br

(3)UFRGS, Faculdade de Agronomia, Dep. de Plantas Forrageiras e Agrometeorologia. Bolsista do PIBIC/CNPq/UFRGS (pontos de demanda). A produção da biomassa de uma pastagem segue um modelo morfogênico determinado para cada uma das espécies constituintes cujo ritmo é condicionado sobretudo pela temperatura. Trata-se, portanto, da integração de dois modelos de funcionamento: um morfogênico, que representa a demanda para crescimento; e um trófico, que representa a oferta de carbono e minerais (Hardy et al., 1984, citados por Medeiros, 1995; Nabinger, 1997a).

Para explicar o crescimento vegetal é necessário analisar o destino do carbono fixado e, particularmente, sua partição entre as partes aéreas e as radiculares e, dentro da parte aérea, a partição em folhas e hastes (Nabinger, 1997b). Quando o crescimento é afetado por uma condição limitante, como, 
por exemplo, insuficiência de $\mathrm{N}$ ou suprimento de água, observa-se que certas partes da planta são priorizadas em detrimento de outras. Da mesma maneira, quando há uma limitação na síntese de assimilados (desfolhação, sombreamento), a partição é modificada para favorecer os órgãos fotossintetizantes (Durand et al., 1991). Existe, então, uma hierarquia na partição de assimilados para a fabricação dos diferentes compartimentos que compõem a biomassa vegetal (folhas, hastes, ramificações e raízes). Na ausência de limitações dos recursos temperatura, água, $\mathrm{N}$ e luz, a prioridade de alocação dos assimilados é para atender à demanda de folhas, hastes, ramificações e finalmente raízes. Na ocorrência de limitação de algum dos fatores que determinam diminuição na oferta de carbono (C), a intensidade de demanda de cada um dos compartimentos é alterada, e a prioridade de alocação de assimilados passa a ser para raízes e reservas (Moraes et al., 1995).

O conhecimento das modificações no consumo de $\mathrm{C}$ e de sua partição entre os principais componentes do rendimento (folhas, hastes, órgãos reprodutivos e raiz) provocadas por limitações ambientais é essencial não apenas para realizar diagnósticos, mas, sobretudo, para construir modelos preditivos. Identificar e quantificar os fatores ambientais que limitam a expressão do potencial das espécies forrageiras possibilita estabelecer a melhor forma de manejar esses fatores, com a finalidade de otimizar a produção animal.

Lemaire \& Salette (1984) propõem um modelo que relaciona o teor de nutriente do tecido vegetal com o acúmulo de matéria seca durante o período de crescimento, para determinar o nível de nutrientes para diferentes pastagens e espécies. Tal modelo é representado pela seguinte expressão:

$\mathrm{Y}=\beta_{0}(\mathrm{X})^{-\beta_{1}}$,

onde $\mathrm{Y}$ é o teor de nutriente da biomassa, $\beta_{0}$ é a disponibilidade inicial do nutriente, $\mathrm{X}$ é o peso da biomassa aérea, e $\beta_{1}$, o coeficiente de diluição do nutriente durante o crescimento.

Para uma comunidade de plantas do grupo metabólico $\mathrm{C}_{3}$ e para plantas isoladas, Greenwood et al. (1990, 1991) e Lemaire \& Gastal (1997) estabeleceram as seguintes expressões em relação ao $\mathrm{N}$ : $\% \mathrm{~N}=4,8(\mathrm{X})^{-0,32}$ e $\% \mathrm{~N}=5,06(\mathrm{X})^{-0,106}$, respectivamente.
O presente trabalho teve como objetivo avaliar o efeito da adubação fosfatada e do tipo de solo sobre a produção e partição da biomassa de Desmodium incanum.

\section{Material e Métodos}

O trabalho foi conduzido em vasos no Departamento de Plantas Forrageiras e Agrometeorologia da Faculdade de Agronomia da Universidade Federal do Rio Grande do Sul, em Porto Alegre, RS, no período de novembro de 1996 a fevereiro de 1997. Os tratamentos consistiram de quatro doses de $\mathrm{P}$, em $\mathrm{mg}$ de $\mathrm{P}_{2} \mathrm{O}_{5} / \mathrm{kg}$ de solo $(\mathrm{P} 1=0$, $\mathrm{P} 2=30, \mathrm{P} 3=60$ e $\mathrm{P} 4=90)$ e dois tipos de solo contrastantes em termos de fertilidade natural e porcentagem de argila: Plintossolo e Latossolo). O Plintossolo pertence à unidade de mapeamento Arroio dos Ratos e foi coletado no Município de Eldorado do Sul de uma área de campo nativo. O Latossolo pertence a uma zona de transição das unidades de mapeamento Erechim, Passo Fundo e Cruz Alta, de acordo com o Levantamento de Reconhecimento dos Solos do Estado do Rio Grande do Sul (Brasil, 1973). Este solo foi coletado no Município de Passo Fundo, de uma área de campo nativo. As doses de $\mathrm{P}$ para o Plintossolo representam em relação à recomendação para a máxima eficiência econômica, estabelecidos pela Comissão de Fertilidade do Solo - RS/SC (1989), 0, 43, 86 e $128 \%$, respectivamente, e para o Latossolo representam $0,33,67$ e $100 \%$, respectivamente. O delineamento experimental utilizado foi o completamente casualizado, num fatorial $4 \times 2$, com quatro repetições. De acordo com os resultados da análise de solo, foram aplicados 2,9 e 6,8 t/ha de calcário dolomítico no Plintossolo e Latossolo, respectivamente, permanecendo os mesmos incubados por 30 dias. Após a correção da acidez, realizou-se a adubação corretiva no Latossolo, aplicando-se $30 \mathrm{mg}$ de $\mathrm{K}_{2} \mathrm{O} / \mathrm{kg}$ de solo e a adubação fosfatada conforme as disponibilidades de $\mathrm{P}$ determinadas pelo tratamento. A semeadura de $D$. incanum foi realizada em vasos de $8 \mathrm{~kg}$, após a escarificação e infecção das sementes com Bradyrhizobium específico.

A evolução da biomassa total e sua partição em parte radicular e parte aérea, e desta, em folhas e hastes, foram avaliadas por meio de amostragens de plantas inteiras, realizadas em quatro épocas durante o período experimental. A primeira remoção de plantas foi realizada no dia $27 / 12$, quando se observou o surgimento das primeiras ramificações secundárias na ramificação primária; a segunda ocorreu no dia 17/1, quando se verificou a emissão das inflorescências; a terceira foi no dia $7 / 2$, no pleno florescimento, e a quarta remoção realizou-se no dia $27 / 2$, 
pois nessa data havia cessado o surgimento de folhas na ramificação primária e em algumas ramificações secundárias. Nas datas da primeira, segunda, terceira e quarta remoção, a soma térmica decorrida após a emergência completa foi de $1.339,80,1.981,30,2.594,50$ e $3.160,30^{\circ} \mathrm{C}$, respectivamente. O material coletado em cada época foi separado em hastes, folhas verdes, folhas senescentes, órgãos florais e raiz. Após a separação, o material foi colocado em estufa a $60^{\circ} \mathrm{C}$ até atingir peso constante e pesado em balança de precisão para estabelecer o rendimento de matéria seca.

Após o término das avaliações, foi feita a análise química de tecido vegetal para determinar o teor total de $\mathrm{Ne}$ $\mathrm{P}$ na matéria seca da parte aérea do material resultante de cada época de remoção de plantas. $\mathrm{O}$ teor de $\mathrm{N}$ do tecido vegetal foi relacionado ao acúmulo de matéria seca durante o período de crescimento, e as regressões obtidas foram comparadas ao modelo proposto por Lemaire \& Gastal (1997), pela seguinte expressão:

$\% \mathrm{~N}=\beta_{0}(\mathrm{MS})^{-\beta_{1}}$,

onde $\% \mathrm{~N}$ é o teor de $\mathrm{N}$ da biomassa, $\beta_{0}$ é a disponibilidade inicial de $\mathrm{N}$, MS é o peso da biomassa da parte aérea, e $\beta_{1}$ o coeficiente de diluição do $\mathrm{N}$ durante o crescimento.

Quanto ao teor de $\mathrm{P}$, na falta de referências, foram apenas estabelecidos os parâmetros $\beta_{0}$ e $\beta_{1}$ em razão das diferentes doses de $\mathrm{P}$ aplicadas nos dois solos

Os efeitos das doses de $\mathrm{P}$ aplicadas e do tipo de solo sobre a produção e partição de matéria seca foram submetidos a análise de variância para dados longitudinais (Castro, 1997). Utilizou-se o nível de significância de $10 \%$ em todas as análises realizadas, tendo em vista a alta variabilidade entre plantas. Para a comparação do efeito do tipo de solo, utilizou-se o teste $\mathrm{F}$, ao passo que para o estudo do efeito das doses de $\mathrm{P}$ aplicadas, ajustou-se a regressão linear. Para a interação P x solo, a complementação da análise foi feita pela comparação de médias pelo teste de Tukey, pois os dados das variáveis não se ajustaram à análise de regressão. Para a interação época x P x solo, ajustou-se a regressão não-linear (exponencial). A significância das equações exponenciais foi verificada por meio do intervalo de confiança dos parâmetros $\beta_{0}$ e $\beta_{1}$

Com relação às equações que relacionam o conteúdo de $\mathrm{N}$ e $\mathrm{P}$ na planta e sua biomassa aérea, a significância da diferença entre as equações obtidas e a equação-modelo foi verificada por meio do intervalo de confiança dos parâmetros $\beta_{0}$ e $\beta_{1}$.

Fez-se o controle local da temperatura e umidade relativa do ar, utilizando-se um termógrafo, onde foram registradas as temperaturas mínimas e máximas diárias.

\section{Resultados e Discussão}

No Plintossolo as doses de P não diferem significativamente da testemunha quanto à produção de matéria seca total e matéria seca da parte aérea (Tabela 1). No Latossolo, a testemunha produz menor quantidade de matéria seca total e matéria seca da parte aérea, em relação às doses de $\mathrm{P}$ aplicadas no solo, as quais não diferem significativamente entre si. O Latossolo, quando adubado com $\mathrm{P}$, é superior ao Plintossolo quanto à produção de matéria seca total e da parte aérea, independentemente da dose de $\mathrm{P}$ aplicada.

Em média, a eficiência do uso do $\mathrm{P}$ aplicado para a produção de matéria seca total é, aproximadamente, três vezes maior no Latossolo. Assim, com $30 \mathrm{mg}$ de $\mathrm{P}_{2} \mathrm{O}_{5}$ aplicado por kg do Latossolo, verifica-se uma eficiência de $166 \mathrm{mg}$ de $\mathrm{MS} / \mathrm{mg}$ de $\mathrm{P}_{2} \mathrm{O}_{5}$, enquanto no Plintossolo essa eficiência limita-se a $45 \mathrm{mg}$ de $\mathrm{MS} / \mathrm{mg}$ de $\mathrm{P}_{2} \mathrm{O}_{5}$. $\mathrm{O}$ aumento nas doses de $\mathrm{P}$ aplicadas reduz essa eficiência, que passa a ser de 96 e $70 \mathrm{mg}$ de $\mathrm{MS} / \mathrm{mg}$ de $\mathrm{P}_{2} \mathrm{O}_{5}$ para os níveis de 60 e $90 \mathrm{mg}$ de $\mathrm{P}_{2} \mathrm{O}_{5}$, respectivamente no Latossolo, enquanto no Plintossolo, situa-se em 35 e $20 \mathrm{mg}$ de $\mathrm{MS} / \mathrm{mg}$ de $\mathrm{P}_{2} \mathrm{O}_{5}$, respectivamente.

As máximas eficiências foram alcançadas com $43 \%$ e $33 \%$ da recomendação para a máxima eficiência econômica no Plintossolo e Latossolo, respectivamente. Cabe salientar que a recomendação é para leguminosas tropicais em geral e em cultura

Tabela 1. Efeito da interação entre doses de fósforo e tipo de solo na produção de matéria seca total e matéria seca da parte aérea de Desmodium incanum. UFRGS, $1996-97^{(1)}$

\begin{tabular}{|c|c|c|c|c|}
\hline \multirow{2}{*}{$\begin{array}{c}\mathrm{P}_{2} \mathrm{O}_{5}(\mathrm{mg} / \mathrm{kg} \\
\text { de solo) }\end{array}$} & \multicolumn{2}{|c|}{ Matéria seca total } & \multicolumn{2}{|c|}{ Matéria seca aérea } \\
\hline & Plintossolo & Latossolo & Plintossolo & Latossolo \\
\hline & \multicolumn{4}{|c|}{ 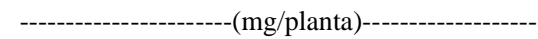 } \\
\hline 0 & $1.563 \mathrm{~B}$ & $1.722 \mathrm{~B}$ & $962 \mathrm{~B}$ & $1.087 \mathrm{~B}$ \\
\hline 30 & 2.913B & $6.695 \mathrm{~A}$ & 1.737B & $4.470 \mathrm{~A}$ \\
\hline 60 & 3.682B & $7.464 \mathrm{~A}$ & 2.357B & $5.003 \mathrm{~A}$ \\
\hline 90 & $3.390 \mathrm{~B}$ & 7.996A & 2.193B & $5.600 \mathrm{~A}$ \\
\hline Média & $2.887 b$ & $5.969 \mathrm{a}$ & $1.812 b$ & $4.040 \mathrm{a}$ \\
\hline
\end{tabular}

${ }^{(1)}$ Médias seguidas de mesma letra, maiúscula na coluna e minúscula na linha, não diferem estatisticamente pelo teste de Tukey a 5\% de probabilidade; os coeficientes de variação em relação à produção de matéria seca total e matéria seca da parte aérea foram, respectivamente, $59,67 \%$ e $59,53 \%$. 
densa. Por isso, pode-se supor que $D$. incanum apresenta exigências diferentes daquelas espécies em que foram obtidas as calibrações para as recomendações, ou algum outro fator não controlado estaria limitando a magnitude da resposta, como, por exemplo, as deficiências de $\mathrm{N}$ e de micronutrientes, visto que praticamente não houve resposta com a aplicação de doses superiores a $30 \mathrm{mg}$ de $\mathrm{P}_{2} \mathrm{O}_{5}$, em ambos os solos.

A produção de matéria seca radicular apresenta uma resposta linear ao $\mathrm{P}$ aplicado $(\mathrm{Y}=564,76+$ $373,53 \mathrm{X}$ com $\left.\mathrm{R}^{2}=0,89\right)$, isto é, estima-se em $373,53 \mathrm{mg}$ o acréscimo na produção de matéria seca de raiz para cada $30 \mathrm{mg}$ de $\mathrm{P}_{2} \mathrm{O}_{5}$ aplicado por $\mathrm{kg}$ de solo. Essa resposta é independente do tipo de solo, ou seja, não há interação entre doses de $\mathrm{P}$ e tipo de solo. A produção média de raízes em razão do efeito solo é significativamente superior no Latossolo ( $1.928 \mathrm{mg} /$ planta) em relação ao Plintossolo (1.075 mg/planta), ou seja, $79 \%$ maior na média das doses de P.

Embora estatisticamente não haja diferença entre as doses de $\mathrm{P}$ aplicadas sobre a relação MSA/MSR, a espécie modifica seu programa morfogênico para adaptar-se às alterações ambientais, isto é, quando ocorre limitação de $\mathrm{P}$ a formação da parte aérea é penalizada em favor da manutenção do sistema radicular, diminuindo a relação MSA/MSR (Tabela 2).

A produção de ramificações secundárias apresenta uma resposta linear $(\mathrm{Y}=842,64+518,13 \mathrm{X}$ com $\left.\mathrm{R}^{2}=0,88\right)$ ao $\mathrm{P}$ aplicado. Logo, o déficit de $\mathrm{P}$ ocasiona uma redução na produção de ramificações secundárias. Conforme Nabinger (1997a), numa situação de recursos escassos, a limitação do crescimento aéreo constitui uma economia, que, traduzindo-se por uma maior utilização do $\mathrm{C}$ no crescimento radicular, permite à população realizar uma melhor exploração dos recursos mais limitantes do meio, no caso o $\mathrm{P}$.

A mesma estratégia adaptativa é utilizada em razão do tipo de solo (Tabela 3). No Plintossolo há uma diminuição na relação MSA/MSR, e as ramificações secundárias participam em $35 \%$ da biomassa total, enquanto no Latossolo elas representam $44 \%$.

Quanto à produção de matéria seca da ramificação primária, independentemente da dose de $\mathrm{P}$ e da fertilidade natural do solo, a espécie mantém a alocação de assimilados para a sua formação, ou seja, tanto na condição do Plintossolo, sem a aplicação de $\mathrm{P}$, como naquela do Latossolo, com o máximo nível de $\mathrm{P}$, a ramificação primária participa em $26 \%$ da biomassa total. Isso evidencia que, numa condição de limitação, a prioridade é, além do desenvolvimento do sistema radicular, a manutenção da ramificação primária.

Durand et al. (1989) também verificaram em alfafa, submetida a estresse hídrico, maior alocação de assimilados para as raízes. Da mesma forma, Morales et al. (1997) relatam que o cornichão penaliza a alocação de assimilados para a formação da parte aérea, sobretudo das ramificações secundárias, em favor das raízes. Os autores observam que as

Tabela 2. Efeito de doses de fósforo na produção de matéria seca das ramificações primárias (MSR1) e na relação matéria seca da parte aérea/matéria seca radicular (MSA/MSR) de Desmodium incanum, em dois tipos de solos. UFRGS, 1996-97(1).

\begin{tabular}{cccc}
\hline \multirow{2}{*}{$\mathrm{P}_{2} \mathrm{O}_{5}$} & \multicolumn{2}{c}{ MSR1 } & \multirow{2}{*}{ MSA/MSR } \\
\cline { 2 - 3 }$(\mathrm{mg} / \mathrm{kg}$ de solo $)$ & Plintossolo & Latossolo & \\
\hline & $-------(\mathrm{mg} /$ planta)------ \\
0 & $413 \mathrm{D}$ & $594 \mathrm{D}$ & \\
30 & $768 \mathrm{CD}$ & $1.366 \mathrm{BC}$ & $2,5 \mathrm{~A}$ \\
60 & $1.079 \mathrm{BCD}$ & $1.598 \mathrm{AB}$ & $3,0 \mathrm{~A}$ \\
90 & $887 \mathrm{CD}$ & $2.090 \mathrm{~A}$ & $3,1 \mathrm{~A}$ \\
\hline Média & $787 \mathrm{~b}$ & $1.412 \mathrm{a}$ \\
\end{tabular}

(1)Médias seguidas de mesma letra, maiúscula na coluna e minúscula na linha, não diferem estatisticamente pelo teste de Tukey a 5\% de probabilidade; os coeficientes de variação em relação a MSR1 e MSA/MSR foram, respectivamente, de $49,11 \%$ e $30,49 \%$

Tabela 3. Efeito do tipo de solo na produção de matéria seca das ramificações secundárias (MSR2) e na relação matéria seca aérea/matéria seca radicular (MSA/MSR) de Desmodium incanum. UFRGS, 1996-97(1)

\begin{tabular}{lcc}
\hline Tratamento & MSR2 (mg/planta) & MSA/MSR \\
\hline Plintossolo & $1.025 \mathrm{~B}$ & $2,6 \mathrm{~B}$ \\
Latossolo & $2.628 \mathrm{~A}$ & $3,1 \mathrm{~A}$ \\
\hline C.V. $(\%)$ & 66,20 & 30,49 \\
\hline
\end{tabular}

(1)Médias seguidas de mesma letra na coluna não diferem estatisticamente pelo teste de Tukey a $5 \%$ de probabilidade. 
raízes passam a representar cerca de $30 \%$ da biomassa quando o solo está com $50 \%$ da sua capacidade de campo contra $22 \%$ na condição não-limitante. A espécie estabelece como prioridade, numa condição de limitação hídrica, a manutenção da haste principal e a formação de raízes. Situação semelhante ocorre em relação ao N. Bélanger et al. (1992), estudando Festuca arundinacea, observaram um aumento na proporção de assimilados alocados para a raiz quando houve limitação do nutriente.

As análises de regressão demonstraram que as equações para a diluição de $\mathrm{N}$ em razão dos tratamentos utilizados diferem da equação modelo proposta por Lemaire \& Gastal (1997).

A disponibilidade inicial de $\mathrm{N}\left(\beta_{0}\right)$ em todos os tratamentos esteve abaixo da estabelecida pela expressão modelo $\left(\beta_{0}=5,06\right)$, ou seja, podemos afirmar que houve limitação de $\mathrm{N}$ nas plantas, certamente decorrente da ineficiência do Rhizobium (Figura 1). Entretanto, quando se utiliza o Latossolo adubado, há maior disponibilidade inicial de $\mathrm{N}\left(\beta_{0}\right)$ para as plantas, possivelmente em virtude da mineralização do $\mathrm{N}$ orgânico e da maior absorção feita em razão de um sistema radicular mais desenvolvido.

$\mathrm{O}$ decréscimo no teor de $\mathrm{N}$ com o acúmulo de matéria seca $\left(\beta_{1}\right)$, que se observa em uma comunidade de plantas, é considerado como uma conseqüência de dois fenômenos, ou seja: na planta individual, o decréscimo pode ser devido ao fato de que à medida que a planta cresce ela contém maior proporção de material estrutural e de reservas que contêm pouco $\mathrm{N}$; na população, esse decréscimo é o resultado da não-uniformidade da distribuição do $\mathrm{N}$ entre folhas, por causa do nível de irradiação recebida no interior do dossel (Caloin \& Yu, 1984; CharlesEdwards et al., 1987). Conforme esses autores, em termos de plantas individuais, a planta pode ser dividida em dois compartimentos: o primeiro é o compartimento metabólico, representado pelo $\mathrm{N}$ associado à fotossíntese e a outros processos metabólicos; e o segundo seria um compartimento estrutural, que consiste de $\mathrm{N}$ em estoque e nos tecidos estruturais. Admitindo-se que o conteúdo de N no compartimento metabólico é muito maior do que no compartimento estrutural, a evolução do conteúdo de $\mathrm{N}$ da planta é determinada pela evolução do tamanho relativo desses dois compartimentos.
Os coeficientes de diluição do N $\left(\beta_{1}\right)$ demonstraram que $D$. incanum rapidamente acumula maior proporção de material estrutural, o que confirma os relatos de Bogdan (1977) e Skerman et al. (1991) a respeito da acelerada lignificação dos ramos dessa espécie.

Salette et al. (1989) afirmam que o modelo de diluição do N pode ser facilmente adaptado para outros minerais. Entretanto, não é possível assumir nenhuma das equações ajustadas para a diluição de $\mathrm{P}$, em razão dos tratamentos, como modelo (Figura 1), visto que, conforme os autores acima citados, somente as curvas de diluição obtidas sob condições nãolimitantes de $\mathrm{N}$ podem ser consideradas como referência, o que provavelmente não se verifica na presente situação.

Segundo Duru \& Thelier-Huche (1997), o modelo de diluição do $\mathrm{P}$ depende da disponibilidade de $\mathrm{N}$, pois existe interação entre a quantidade de $\mathrm{N}$ aplicado e a concentração de $\mathrm{P}$ na pastagem. Os autores propõem um modelo representado pela seguinte expressão: $\% \mathrm{P}=0,24(\% \mathrm{~N})^{0,64}$

Em relação ao modelo proposto por Lemaire \& Gastal (1997), podemos considerar que talvez fosse relevante investigar se a disponibilidade inicial de $\mathrm{N}$ estabelecida pelo modelo ( $\left.\beta_{0}\right)$ é realmente a necessária para obter-se o desenvolvimento potencial de D. incanum. Para solucionar tal questão, poder-seia, utilizando $\mathrm{N}$ mineral, estabelecer curvas de diluição do N, e assim, comparar os coeficientes obtidos com os propostos por estes pesquisadores. Dessa forma, verificar-se-ia a validade do modelo para a espécie em estudo.

Ao admitir-se que o modelo geral de diluição de N proposto por Lemaire \& Gastal (1997) não é válido para $D$. incanum, pode-se propor como modelo específico para o $\mathrm{N}$, aquele obtido quando, ao aumentar-se a dose de $\mathrm{P}$ no solo de melhor fertilidade natural, o modelo não mais varia, ou seja, na dose de 60 e $90 \mathrm{mg}$ de $\mathrm{P}_{2} \mathrm{O}_{5} / \mathrm{kg}$ de solo. Para essas doses tem-se $\beta_{0}=3,45$ e $\beta_{1}=0,22$.

A proposição de um modelo diferente para D. incanum sustenta-se na alta proporção de estolhos e outras estruturas não incluídas na parte aérea e que, por serem mais permanentes, alocam uma alta proporção do N para a formação dos componentes estruturais. 

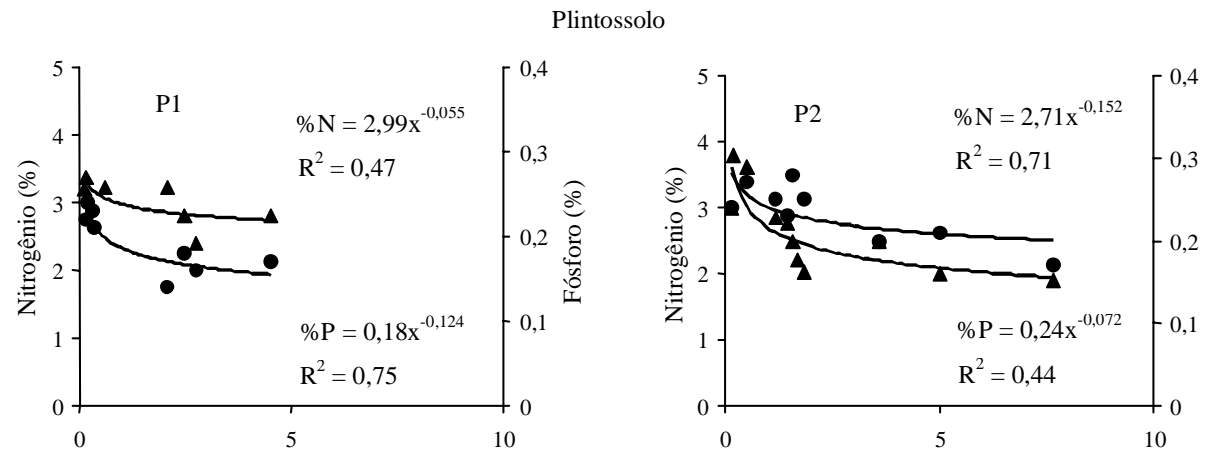

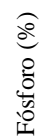
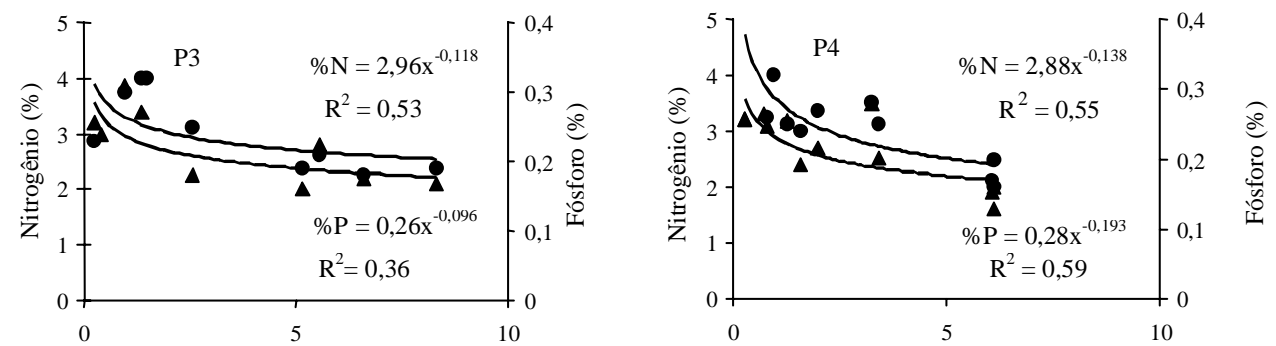

Latossolo
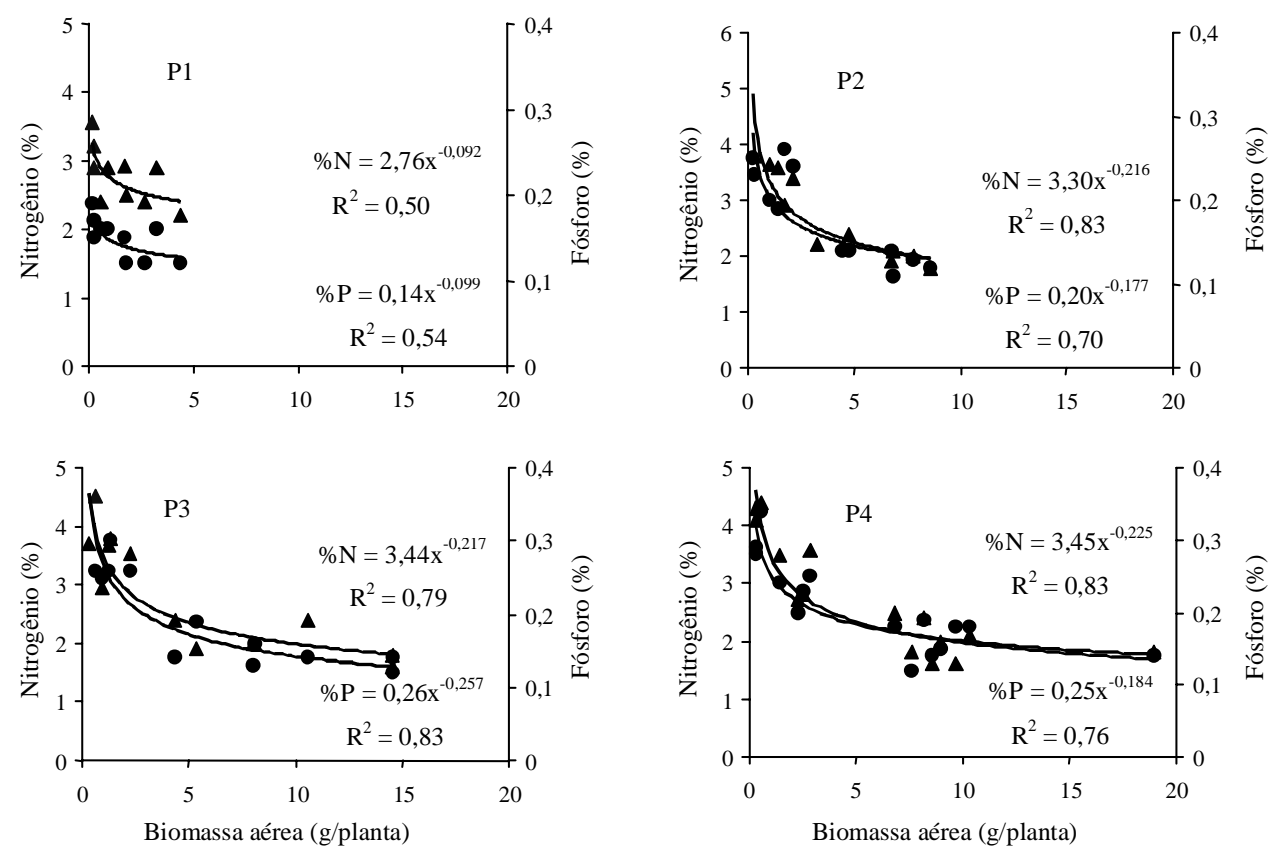

$\Delta$ Nitrogênio

- Fósforo

Figura 1. Curva de diluição entre o teor de nitrogênio $(\mathrm{N})$ e de fósforo $(\mathrm{P})$, e a biomassa aérea, de Desmodium incanum, em razão de doses de fósforo ( $\mathrm{P}_{1}: 0, \mathrm{P}_{2}: 30, \mathrm{P}_{3}: 60$ e $\mathrm{P}_{4}: 90 \mathrm{mg}$ de $\mathrm{P}_{2} \mathrm{O}_{5} / \mathrm{kg}$ de solo) aplicadas no Plintossolo e no Latossolo. UFRGS, 1996-97. 
A utilização do modelo de diluição permite identificar, além de deficiências nutricionais, a eficiência do Rhizobium na fixação simbiótica, principalmente nas espécies que ainda não possuem Rhizobium específico, ou que estão sendo pesquisadas, para serem selecionadas estirpes mais eficientes.

\section{Conclusões}

1. A resposta de Desmodium incanum à adição de fósforo é superior no Latossolo.

2. A deficiência de fósforo e a menor fertilidade natural do solo causam redução na produção de ramificações secundárias.

\section{Referências}

BÉLANGER, G. ; GASTAL, F.; WAREMBOURG, F. The effects of nitrogen fertilization and the growing season on carbon partitioning in sward of tall fescue. Annals of Botany, London, v. 70, p. 239-244, 1992.

BOGDAN, A. V. Tropical pasture and fodder plants: grasses and legumes. London : Longman, 1977. 475 p. (Tropical Agriculture Series)

BRASIL. Ministério da Agricultura. Departamento Nacional de Pesquisa Agropecuária. Divisão de Pesquisa Pedológica. Levantamento de reconhecimento dos solos do Estado do Rio Grande do Sul. Recife, 1973. 431 p. (DNPEA. Boletim Técnico, 30).

CALOIN, M.; YU, O. Analysis of the time course of change in nitrogen content in Dactylis glomerata L. using a model of plant growth. Annals of Botany, London, v. 54, p. 69-76, 1984.

CASTRO, S. M. de J. A metodologia de análise de dados longitudinais. Porto Alegre : UFRGS, 1997. $119 \mathrm{p}$. Monografia de bacharelado em Estatística.

CHARLES-EDWARDS, D. A.; STUTZEL, H.; FERRARIS, R.; BEECH, D. F. An analysis of spatial variation in the nitrogen content of leaves from different horizons within a canopy. Annals of Botany, London, v. 60 , p. $421-426,1987$

COMISSÃO DE FERTILIDADE DO SOLO - RS/SC (Passo Fundo, RS). Recomendação de adubação e calagem para os estados do Rio Grande do Sul e Santa Catarina. 2.ed. Passo Fundo, 1989. 128 p.

DURAND, J. L.; LEMAIRE, G.; GOSSE, G.; CHARTIER, $\mathrm{M}$. Analyse de la conversion de l'énergie solaire en matière sèche par un peuplement de luzerne (Medicago sativa L.) soumis à un déficit hydrique. Agronomie, Paris, v. 9 , p. 599-607, 1989.

DURAND, J. L.; VARLET-GRANCHER, C.; LEMAIRE, G.; GASTAL, F.; MOULIA, B. Carbon partitioning in forage crops. Acta Biotheoretica, Dordrecht, v. 39, p. 213-224, 1991.

DURU, M.; THELIER-HUCHE, L. N and P-K status of herbage: use for diagnosis of grasslands. In: LES COLLOQUES, INSTITUT NATIONAL DE LA RECHERCHE AGRONOMIQUE, 82., 1995, Poitiers. Diagnostic procedures for crop $\mathbf{N}$ management. Paris : INRA, 1997. p. 125-137.

GREENWOOD, D. J.; GASTAL, F.; LEMAIRE, G.; DRAYCOTT, A.; MILLARD, P.; NEETESON, J. J. Growth rate and $\% \mathrm{~N}$ of field grown crops: theory and experiments. Annals of Botany, London, v. 67, p. 181-190, 1991

GREENWOOD, D. J.; LEMAIRE, G.; GOSSE, G.; CRUZ, P.; DRAYCOTT, A.; NEETESON, J. J. Decline in percentage $\mathrm{N}_{\text {of }} \mathrm{C}_{3}$ and $\mathrm{C}_{4}$ crops with increasing plant mass. Annals of Botany, London, v. 66, p. 425-436, 1990.

LEMAIRE, G.; GASTAL, F. N uptake and distribution in plant canopies. In: DIAGNOSIS of the nitrogen status in crops. Paris : INRA, 1997. p. 3-43

LEMAIRE, G.; SALETTE, J. Relation entre la dynamique de croissance e dynamique de prélèvement d'azote pour un peuplement de graminées fourragères. I. Études de l'effect du millieu. Agronomie, Paris, v. 4, n. 5 , p. $423-430,1984$

MEDEIROS, R. B. de. Modelos de semeadura de alfafa (Medicago sativa $\mathrm{L}$.) e suas relações com o desenvolvimento vegetativo e reprodutivo. Porto Alegre : UFRGS, 1995. 236 p. Tese de Doutorado.

MORAES, A.; MARASCHIN, G. E.; NABINGER, C. Pastagens nos ecossistemas de clima sub-tropical: pesquisas para o desenvolvimento sustentável. In: SIMPÓSIO SOBRE PASTAGENS NOS ECOSSISTEMAS BRASILEIROS, 32., 1995, Brasília. Anais... Brasília : Sociedade Brasileira de Zootecnia, 1995. p. 147-200.

MORALES, A. S. de.; NABINGER, C.; ROSA, L. M.; MARASCHIN, G. E. Efeito da disponibilidade hídrica sobre a morfogênese e repartição de assimilados em L. corniculatus L. cv. São Gabriel. In: REUNIÃO ANUAL DA SOCIEDADE BRASILEIRA DE ZOOTECNIA, 34 ., 1997, Juiz de Fora. Anais... Juiz de Fora : Sociedade Brasileira de Zootecnia, 1997. p. 124-126. 
NABINGER, C. Eficiência do uso de pastagens: disponibilidade e perdas de forragem. In: SIMPÓSIO SOBRE MANEJO DAPASTAGEM, 14., 1997, Piracicaba. Anais. Piracicaba : FEALQ, 1997a. p. 213-251

NABINGER, C. Princípios da exploração intensiva de pastagens. In: SIMPÓSIO SOBRE MANEJO DA PASTAGEM, 13., 1996, Piracicaba. Anais... Piracicaba : FEALQ, 1997b. p. 15-95
SALETTE, J.; HUCHÉ, L.; LEMAIRE, G. Modelling nitrogen and mineral nutrient uptake by a grass sward: short term studies during the sward growth. In INTERNATIONAL GRASSLAND CONGRESS, 16. 1989, Nice. Proceedings... Nice : The French Grassland Society, 1989. p. 61-62.

SKERMAN, P. J.; CAMERON, D. G.; RIVEROS, F. Leguminosas forrajeras tropicales. Roma : FAO, 1991. $707 \mathrm{p}$. 